\title{
From Mathematical Physics to Analysis: A Walk in Barry Simon's Mathematical Garden, II
}

Fritz Gesztesy

Editor's Note: This is a continuation of the August feature in honor of Barry Simon on the occasion of his 2016 AMS Leroy P. Steele Prize for Lifetime Achievement and his seventieth birthday conference August 28-September 1, 2016. The authors of Part I were P. A. Deift, J. Fröhlich, E. M. Harrell, M. Reed, L. Rosen, and F. Gesztesy, who coordinated Parts I and II.

\section{Joseph (Yosi) Avron}

\section{Barry and Pythagoras \\ Admiration}

I passionately admired Barry in the years that shaped me: he seemed to know everything that was worth knowing, be it math, physics, history, or literature; he could think faster than anyone else I knew; he could write mathematics

\section{Barry [is] bigger than life} so it read like beautiful poetry, and he did it effortlessly; he was a wonderful teacher who could give a perfectly organized proof of any theorem on the spur of the moment and he could multitask like a superhuman being. Barry was bigger than life. He was my idol and has since been an important part of my life.

\section{Of Walks and Traces}

Barry used to visit Israel regularly. He always set up base at the Hebrew University in Jerusalem and came for a day or two to give a seminar at the Technion. Barry made his itinerary early, which meant that I had plenty of time to get ready for his visit, which really meant that I had plenty of time to worry what worthwhile observation I had to impress Barry with. Barry's visits were like my annual driving tests: if Barry simply shrugged and lost interest,

Fritz Gesztesy is Jean \& Ralph Storm Chair of Mathematics at Baylor University. His email address is Fritz_Gesztesy@baylor . edu.

Joseph (Yosi) Avron is professor of physics at the Technion, Haifa. His email address is avron@physics . technion . ac. $i 1$.

For permission to reprint this article, please contact:

reprint-permission@ams.org.

DOI: http://dx.doi.org/10.1090/noti1412

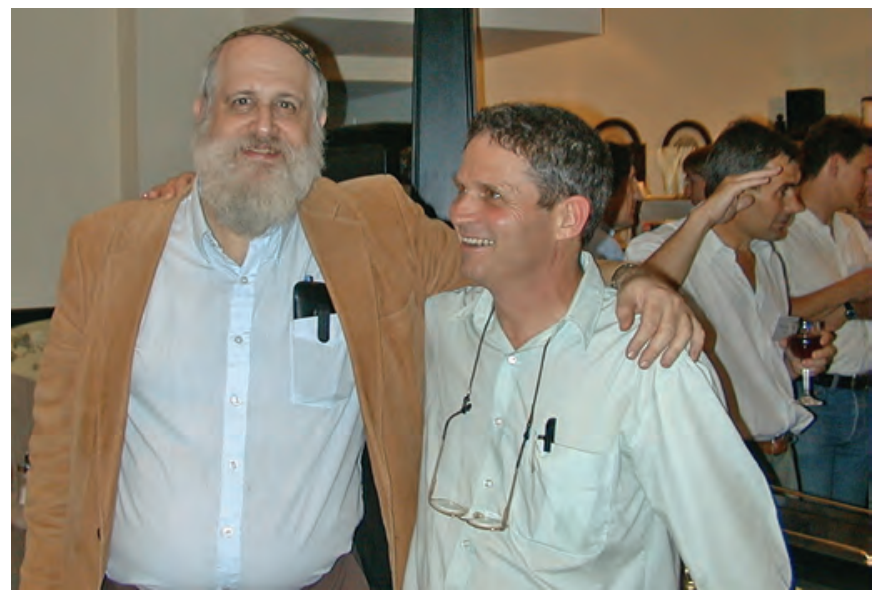

Barry and Yosi, QMath7, Prague, 1998.

this meant that I flunked. Here is the story of a visit that eventually led to a joint paper.

Sometime in 1990, following Barry's seminar at the Technion, we were strolling through campus. This time I came prepared. Ruedi Seiler and I were trying to understand Jean Bellissard's noncommutative geometry of the quantum Hall effect, where comparison of infinitedimensional projections plays a role. I told Barry what I thought was an amusing identity about a pair of finite dimensional projections:

$$
\operatorname{Tr}(P-Q)=\operatorname{Tr}(P-Q)^{3} .
$$

You can verify equation (1) using $P^{2}=P$ and $Q^{2}=Q$ and the cyclicity of the trace. But this does not really explain why the relation is true.

Memories are fragmented and treacherous. I cannot tell today if I found the trace identity on my own or if I learned it and conveniently forgot who taught it to me. Bellissard taught me how many wonderful facts about 
traces and identities similar to the trace identity play a role in his theory of the quantum Hall effect [2], so it is possible that he taught me this identity and I simply forgot.

\section{Anticommutative Pythagoras}

The following day Barry showed me two identities involving a pair of orthogonal projections that in one fell swoop explained the trace identity and put it in a much broader context. My favorite mnemonic for these identities is anticommutative Pythagoras

$$
C^{2}+S^{2}=1, \quad C S+S C=0,
$$

where the "cosine" and "sine" are differences of projections:

$$
C=P-Q, \quad S=P_{\perp}-Q=1-P-Q .
$$

\section{Supersymmetry}

Here is how equations (1) and (2) are related: Suppose $\lambda \neq \pm 1$ is an eigenvalue of (the self-adjoint) $C$ :

$$
C|\psi\rangle=\lambda|\psi\rangle \text {. }
$$

Then $-\lambda$ is also an eigenvalue of $C$, with eigenvector $|\phi\rangle=S|\psi\rangle$. This follows from

$$
C|\phi\rangle=C S|\psi\rangle=-S C|\psi\rangle=-\lambda S|\psi\rangle=-\lambda|\phi\rangle .
$$

The proviso $\lambda \neq \pm 1$ comes about because one needs to make sure $|\phi\rangle \neq 0$. Indeed, since $P, Q$ are orthogonal projections, $S=S^{*}$, and

$$
\begin{aligned}
\langle\phi \mid \phi\rangle & =\left\langle\psi\left|S^{*} S\right| \psi\right\rangle=\left\langle\psi\left|S^{2}\right| \psi\right\rangle=\left\langle\psi\left|1-C^{2}\right| \psi\right\rangle \\
& =\left(1-\lambda^{2}\right)\langle\psi \mid \psi\rangle .
\end{aligned}
$$

It follows that if $C$ is trace class, then the trace of all odd powers of $C$ coincide:

$$
\begin{aligned}
& \operatorname{Tr}(P-Q)=\operatorname{Tr}(P-Q)^{2 n+1} \\
& \quad=\operatorname{dim} \operatorname{ker}(C-1)-\operatorname{dim} \operatorname{ker}(C+1) \in \mathbb{Z} .
\end{aligned}
$$

This is illustrated in Figure 1.

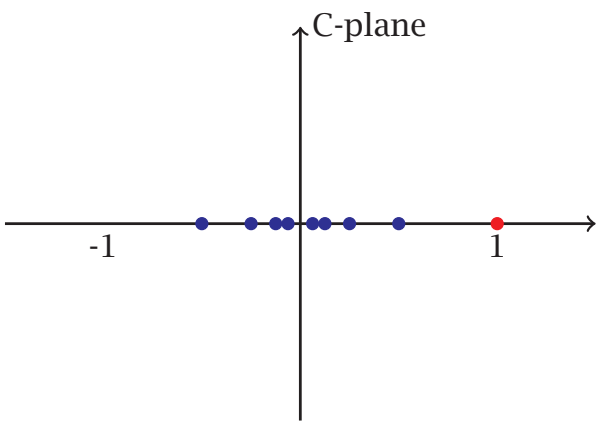

Figure 1. The spectrum of $C$ : The paired eigenvalues $-1< \pm \lambda_{j}<1$ are marked in blue. The eigenvalue at 1 is unpaired and is marked in red.

If $P-Q$ is compact, then the right-hand side of equation (4) gives a natural "regularization" of the trace and shows that it is always an integer.

\section{The Quantum Hall Effect}

Pairs of projections play a role in the theory of the quantum Hall effect. Let me only point out how physics and math shed light on each other in the case of equation (1).

For three projections, $P, Q, R$, the trace identity implies that

$$
\operatorname{Tr}(P-Q)^{3}=\operatorname{Tr}(P-R)^{3}+\operatorname{Tr}(R-Q)^{3},
$$

which follows from

$$
\operatorname{Tr}(P-Q)=\operatorname{Tr}(P-R)+\operatorname{Tr}(R-Q) .
$$

This makes one wonder: Why should cubic powers of differences of projection behave linearly upon tracing?

A physical insight into the linearity comes from interpretation of $\operatorname{Tr}(P-Q)^{3}$ as the Hall conductance. The linearity of equation (5) may then be viewed as a version of Ohm's law of the additivity of conductances.

\section{Slow Script}

Barry had the reputation of being the fastest pen in the West. So, writing these memoirs, I was actually surprised to find out that our paper [1] came out only four years later. It was written during one of Barry's subsequent visits to Israel in his tiny cramped office at the Einstein Institute at the Hebrew University.

\section{References}

[1] J. Avron, R. Seiler, and B. Simon, The index of a pair of projections, J. Funct. Anal. 120 (1994), 220-237. MR1262254

[2] J. BELlissaRD, A. VAN ElST, and H. Schulz-BALDES, The noncommutative geometry of the quantum Hall effect, J. Math. Phys. 35 (1994), 5373-5451. MR1295473

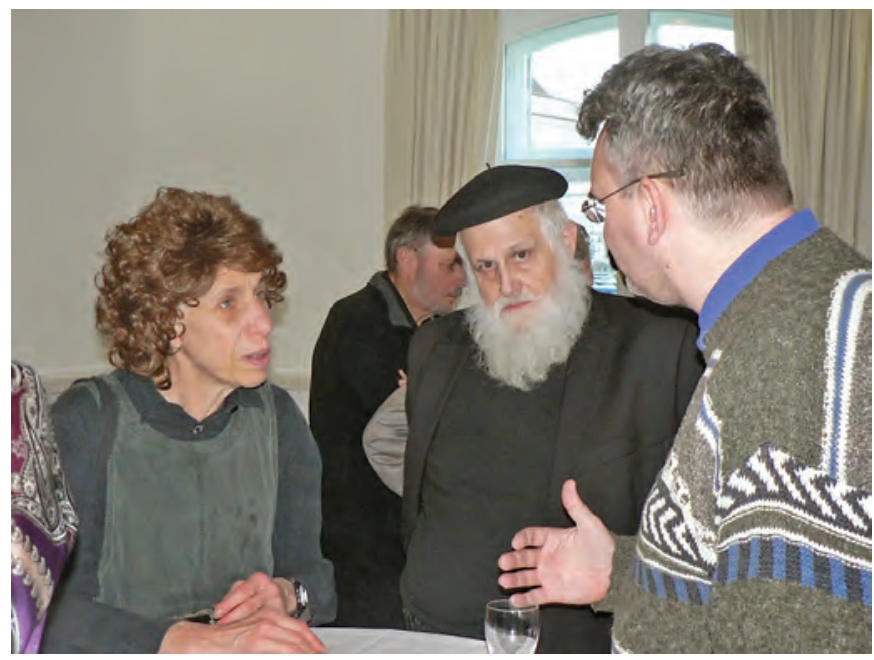

Martha and Barry Simon, Ludwig-MaximiliansUniversity of Munich, 2014. 


\section{Svetlana Jitomirskaya}

\section{Quasiperiodic Schrödinger Operators}

"In many years, flu sweeps the world. The actual strain varies from year to year; some years it has been Hong Kong flu, some years swine flu. In 1981, it "In many years, flu was the almost perisweeps the odic flu!" So starts Barry Simon's paper world...In 1981, it was the almost periodic flu!"
Meanwhile, Avron and Simon noted that earlier work of Gordon implied that this model also provides an easy example of a singular continuous spectrum. No wonder that the "flu" started spreading also in the math world, where of course it was only natural to consider the more general class of almost periodic operators.

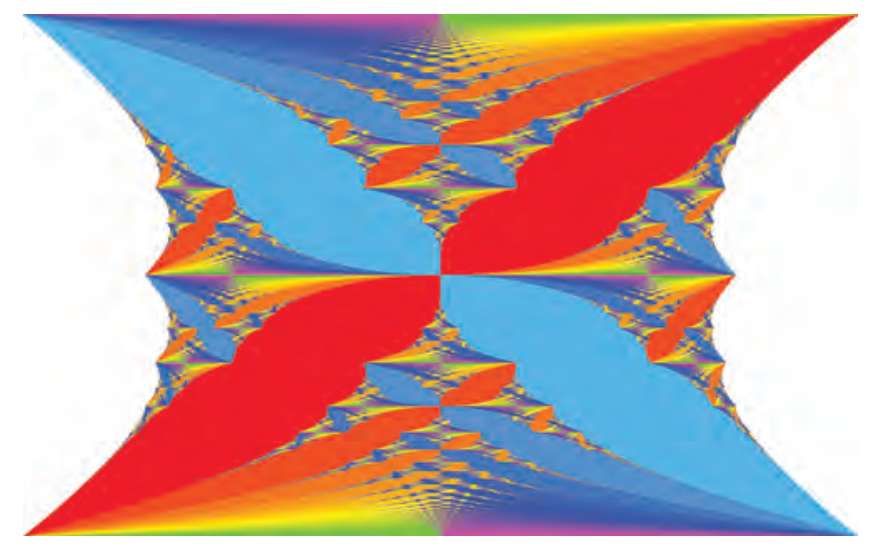

Figure 2. The colored Hofstadter butterfly.

Over the years, the field has seen a number of fundamental advances by many contributors. Sinai's and Fröhlich-Spencer-Wittwer's KAMs, Helffer-Sjóstrand's semiclassical analysis, Eliasson's reducibility/perfect analytic KAM, Bourgain's analytic revolution that made nonperturbative methods robust and allowed them to go multidimensional, deep results by Goldstein-Schlag and others all kept adding to the excitement. Then there were further physics discoveries making almost periodic models, particularly the almost Mathieu operator, relevant in new contexts. The most remarkable of those was the theory of Thouless et al. that explained the quantization of charge transport in the integer quantum Hall effect-a Nobel Prize winning discovery by von Klitzing in 1980as connected with certain topological invariants (Chern numbers). Central to their theory is the use of the almost Mathieu operator. Moreover, predictions of Thouless et al. were verified experimentally by Albrecht, von Klitzing, et al. in 2001. Three further Nobel Prizes-quasicrystals, graphene, and topological insulators-were also linked to this field, playing a role in the unceasing spread of the "flu."

Barry's flu paper, along with his further papers from the 1980s, besides making some fundamental contributions, defined the foundations of this field in a way that made it very appealing for new students to come in. In fact, that's the way the field, despite many major advances, is seen to this day, with Chapter 9 of Barry's 1982 Thurnau Summer School lecture notes [1] still being the best quick introduction to the subject.

To give but one small illustration of how Barry's work contributed to the worldwide flu spread, one can look at Moscow in the 1980s. My advisor, Yasha Sinai, had a significant preprint problem. With no office or even table space at the Moscow State University, he kept all the preprints people had been sending him from all over the world on a big desk in his two-room apartment. The preprints were piling up, so by the late 1980s, when I was
Svetlana Jitomirskaya is professor of mathematics at the University of California at Irvine. Her email address is szhitomi@math.uci .edu. 


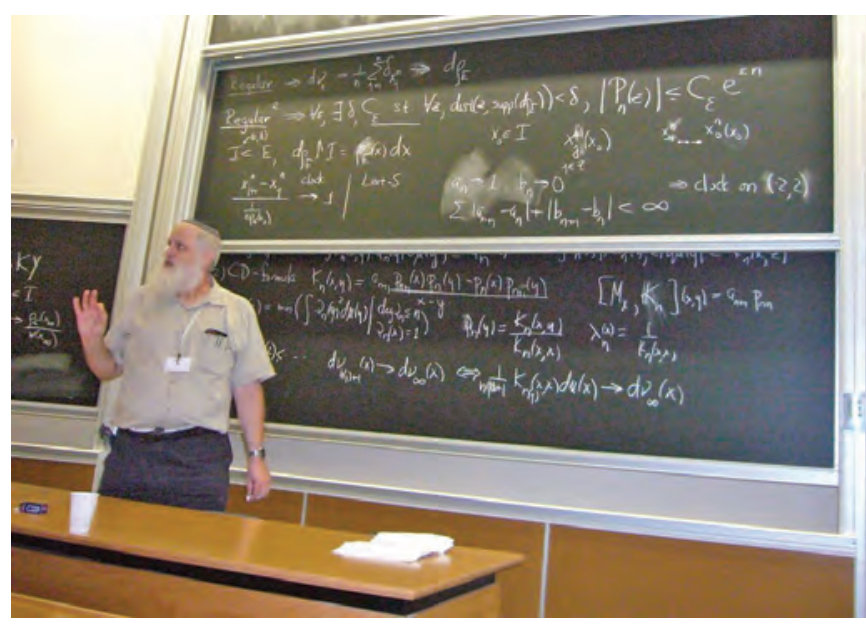

Barry Simon, Marseille, 2007.

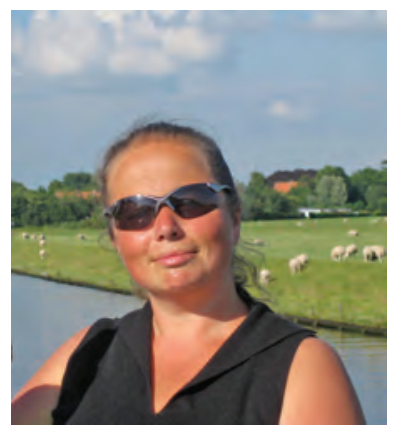

his student, there was no space at all left to work on that desk, and Sinai was using a tiny bureau for writing. At some point he declared that he would throw away an old preprint for every new one he received. However some preprints were too precious to throw away, especially since it was almost impossible to get ahold of most articles by other means. Then Sinai had the idea to give thematic bunches of preprints to his various students. That's how I got ahold of all of Barry's quasiperiodic preprints from the 1980s. My role was to be a librarian for the bunch: I had them catalogued and was checking them out for two weeks at a time to various readers (and following up with the undisciplined ones who tried to hold onto them for a longer period). In Moscow it was still pre-Xerox time, but the flu found its way through the Iron Curtain nevertheless. The popularity of those preprints led to my knowing them very well, so that I could check out the one with the requested fact rather than the whole bunch. Also, I spent most of my time in graduate school as a stay-at-home mom, which gave me more time alone with those preprints. They quickly got me hooked, both by the subject and also the clarity, elegance, and freshness of Barry's writings. The never-boring style required a level of mental workout that seemed just right. The style seemed so "textbook classic" to me that when I came to UCI in the early 1990s and Abel Klein offered to take me along to Caltech "to meet Barry Simon," my first reaction was, literally, "Is he still alive?"

After the 1980s, aside from the Avron-van MoucheSimon paper that came $\varepsilon$-close to proving one of the AubryAndre conjectures, our joint work on singular continuous spectra, and important results with Gesztesy and Last that came as corollaries of more general developments, Barry seemingly got cured himself and moved on to other areas, yet the damage to the world was already done.
Arguably, even more important for the spread was Barry's fifteen problems paper [4]. There he gave a list of fifteen (according to the title, but in reality thirtyfive) important problems in mathematical physics, where, along with most fundamental questions such as "existence of crystals," he threw in the mix a couple of problems on the spectral theory of the almost Mathieu operator, listed as conjectures. Well, is there anything that could better entice a talented young person to enter the field than an attractive and accessible conjecture by Barry Simon appearing in a list like that? The answer is "Of course! It is a wrong such conjecture by Barry Simon." Indeed, that's how Yoram Last entered the area, disproving in his thesis written under the direction of Yosi Avron a wrong part of the almost Mathieu conjecture. Despite a lot of progress in the 1990s, some of the correct parts were not yet fully solved, and then Barry did something even bolder. In his list of (now only) fifteen problems in "Schrödinger operators in the twenty-first century" [5], Barry devoted three(!) to some of the more delicate remaining issues in the spectral theory of the almost Mathieu operator. This did not go unnoticed by the new young generation. A fresh PhD, J. Puig, solved an almost-everywhere version of the Ten Martini problem, with the enticing name coined, of course, by Barry. At about the same time, another fresh $\mathrm{PhD}$, Artur Avila, set out to fully solve all three almost Mathieu problems of [5], which he methodically did, some with coauthors. This got him infected enough to devote his Fields Medal talk in 2014 entirely to the field of quasiperiodic operators, despite having other accomplishments.

It is particularly remarkable that two of the problems were unresolved only for zero measure sets of parameters, and including those in the list of fifteen for the twentyfirst century highlighted the fact that the field was moving from measure theory/probability towards analytic number theory, with recent advances making it possible to seek very precise information for all values of parameters. This defined a significant trend in the later development: interplay of spectral theory with arithmetics, sometimes important only for the proofs, ${ }^{1}$ but at times showing fascinating arithmetic phase transitions.

For example, one of the Aubry-Andre conjectures predicted a metal insulator transition for (6): absolutely continuous spectrum for $\lambda<1$ and pure point for $\lambda>1$, based on Fourier-type duality of the family (6) between these two regions, called subcritical and supercritical. Barry's corrected conjecture acknowledged the possibility of the singular continuous spectrum and dependence on the arithmetics. It turns out that as far as the subcritical regime $\lambda<1$ goes, Aubry and Andre were right after all, with the final result obtained by Avila in 2008, and this is a reflection of a more general phenomenon better understood in the framework of Avila's global theory and almost reducibility theorem. However, in the supercritical

${ }^{1}$ For example, the celebrated Ten Martini proof was dealing, after Puig's work, only with the remaining measure zero set of nonDiophantine frequencies, and while the end result is arithmetics independent, the proof centers around delicate arithmetic issues. 
regime the dependence on the arithmetics is even more subtle than originally anticipated. Namely, let $\frac{p_{n}}{q_{n}}$ be the continued fraction approximants of $\alpha \in \mathbb{R} \backslash \mathbb{Q}$. For any $\alpha, \theta$ we define $\beta(\alpha), \delta(\alpha, \theta) \in[0, \infty]$ as

$$
\begin{aligned}
& \beta=\beta(\alpha)=\limsup _{n \rightarrow \infty} \frac{\ln q_{n+1}}{q_{n}}, \\
& \delta=\delta(\alpha, \theta)=\limsup _{n \rightarrow \infty} \frac{-\ln |||2 \theta+n \alpha|||}{|n|} .
\end{aligned}
$$

We say that $\alpha$ is Diophantine if $\beta(\alpha)=0$ and that $\theta$ is $\alpha$-Diophantine if $\delta(\alpha, \theta)=0$. Lebesgue almost all $\alpha, \theta$ are Diophantine. Then we have the following pair of transition results [2]:

(1) For Diophantine $\alpha$ and any $\theta$ the spectrum undergoes a transition from purely singular continuous for $1<\lambda<e^{\delta}$ to pure point for $\lambda>e^{\delta}$.

(2) For $\alpha$-Diophantine $\theta$ and any $\alpha$ the spectrum undergoes a transition from purely singular continuous for $1<\lambda<e^{\beta}$ to pure point for $\lambda>$ $e^{\beta}$.

This confirms a conjecture I made in 1994, also recently partially solved by Avila, You, and Zhou. Here the conjecture-making was definitely just an attempt to emulate Barry. In fact, this particular conjecture was partly motivated by Barry's work on the Maryland model, where he was the first to go so deep into the interplay between the spectral theory and arithmetic. That program was finally completed recently in our paper with Liu, presenting a full description of spectral transitions for all values of parameters. Moreover, [2] contains the description of exact asymptotics of corresponding eigenfunctions and transfermatrices, opening up a number of exciting possibilities for further analysis.

The almost periodic flu is currently in full strength, and new vistas - and new conjectures-constantly keep coming. With his fundamental contributions and the many cases in which he was responsible for the original infection, Barry deserves significant blame!

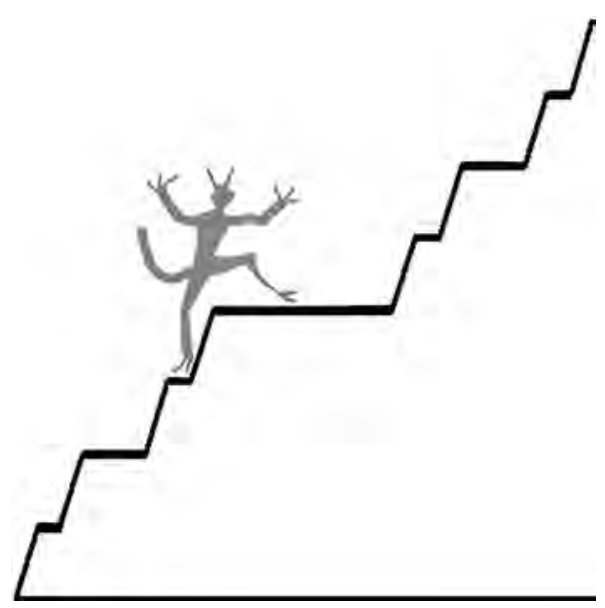

The Cantor function, or Devil's Staircase.

\section{References}

[1] H. Cycon, R. Froese, W. Kirsch, and B. Simon, Schrödinger Operators with Application to Quantum Mechanics and Global Geometry, Texts and Monographs in Physics, Springer-Verlag, Berlin, 1987. MR883643

[2] S. JITOMIRSKAYA and W. LIU, Universal hierarchial structure of quasiperiodic eigenfunctions, preprint, 2016.

[3] B. Simon, Almost periodic Schrödinger operators: A review, Adv. in Applied Math. 3 (1982), 463-490. MR682631

[4] _ Fifteen problems in mathematical physics, in Perspectives in Mathematics: Anniversary of Oberwolfach 1984, W. Jäger, Jürgen Moser, and R. Remmert (eds.), Birkhäuser, Boston, 1984, pp. 423-454. MR779685

[5] _ Schrödinger operators in the twenty-first century, in Mathematical Physics 2000, A. Fokas, A. Grigoryan, T. Kibble, and B. Zegarlinski (eds.), Imperial College Press, London, 2000, pp. 283-288. MR1773049

\section{David Damanik}

\section{Mathematical Physics at Caltech around the Turn of the Century; from Schrödinger Operators with Exotic Spectra to Orthogonal Polynomials on the Unit Circle}

Like many outstanding mathematicians, Barry has changed his research area focus from time to time. This was on display at his sixtieth birthday conference at Caltech in 2006, where each of the five

...his moving from days was devoted exotic spectrato to one major area exotic spectrato to which he has OPUC was most made substantial conOPUC was most tributions. Each day natural and corresponded roughly to one decade of work; perhaps almost the talks on the fourth unavoidable Schrödinger operators Schrödinger operators
with exotic spectra, which were the focus of much of Barry's research in the 1990s, while the talks on the fifth day presented work on orthogonal polynomials, an area to which Barry devoted most of his attention in the early 2000s.

I was extremely fortunate to be a member of Barry's research group for most of the period 1996-2006. I had joined his group primarily due to my interest in exotic spectra, but seeing his transformation into an OPUC (orthogonal polynomials on the unit circle) guru gave me a front-row experience of witnessing something special. The ease and speed with which Barry absorbed an enormous amount of material and turned into one of history's foremost experts in an area which had initially been quite foreign to him was truly amazing.

However, in hindsight his moving from exotic spectra to OPUC was most natural and perhaps almost unavoidable. Let me explain...

David Damanik is Robert L. Moody Sr. Chair in Mathematics at Rice University. His email address is damanik@rice.edu. 
Barry had for a long time been interested in the mathematics of quantum mechanics and, in particular, the spectral analysis of Schrödinger operators $H=-\Delta+V$. The most basic questions here concern the spectrum of $H$, or the allowed energies of the system, and the spectral measures of $H$, from which one may glean information about the long-time behavior of the solutions of the Schrödinger equation $i \partial_{t} \psi=H \psi$. In the good old days, researchers in this field analyzed atomic models, where $V$ vanishes reasonably rapidly at infinity, or crystalline models, where $V$ has translation symmetries forming a full-rank lattice. In both cases the spectrum, which is a subset of the real line, will consist of nondegenerate intervals plus possibly some isolated points outside these intervals. The spectral measures, which are supported by the spectrum, will in these cases have an absolutely continuous component, plus possibly some point masses. The latter will sit at the isolated points of the spectrum, but they may also sit inside the nondegenerate intervals. While the standard decomposition of a measure on the real line will also allow for a singular continuous component, in the early days no nontrivial singular continuous components were known to occur for spectral measures of Schrödinger operators $H$ with "reasonable" potentials $V$, and quite a bit of effort was devoted to actually proving that they indeed do not occur under suitable assumptions on $V$.

Both of these paradigms were severely challenged due to discoveries in the 1970 s and 1980s. Spectra containing neither nondegenerate intervals nor isolated points (Cantor sets) were discovered in the context of almost periodic $V$, and examples of potentials $V$ were found for which there actually did occur singular continuous spectral measures. The early results in these directions were obtained for suitable examples. However, both phenomena were understood at a much deeper level in the 1990s, and Barry was at the center of many of these developments. In fact, both phenomena turned out to be generic in a suitable sense, and Barry contributed key results. For example, in a series of seven papers in the 1990s with a variety of coauthors, Barry studied the occurrence of singular continuous measures in spectral theory, discussing mechanisms leading to them, as well as genericity questions about the applicability of these mechanisms. It was this series of papers that drew me to Barry's work and caused me to move from Germany to sunny Southern California.

One setting in which a strong effort was made in the 1990s to clarify precisely which assumptions preclude or allow certain spectral phenomena was the case of decaying potentials. To be specific, consider Schrödinger operators $H$ on the half-line, that is, in the Hilbert space $L^{2}(0, \infty)$, for which the potential $V$ is small at infinity. For example, one may assume a power-law decay condition $|V(x)| \leq C(1+|x|)^{-\gamma}, \gamma>0$, or an integrability condition $V \in L^{p}(0, \infty), p \geq 1$. Under these assumptions the spectrum of $H$ will consist of the half-line $[0, \infty)$, plus possibly some isolated points below zero that can accumulate only at zero. Thus the shape of the spectrum is classical in the sense described above. It is not clear, however, whether the spectral measures are classical as

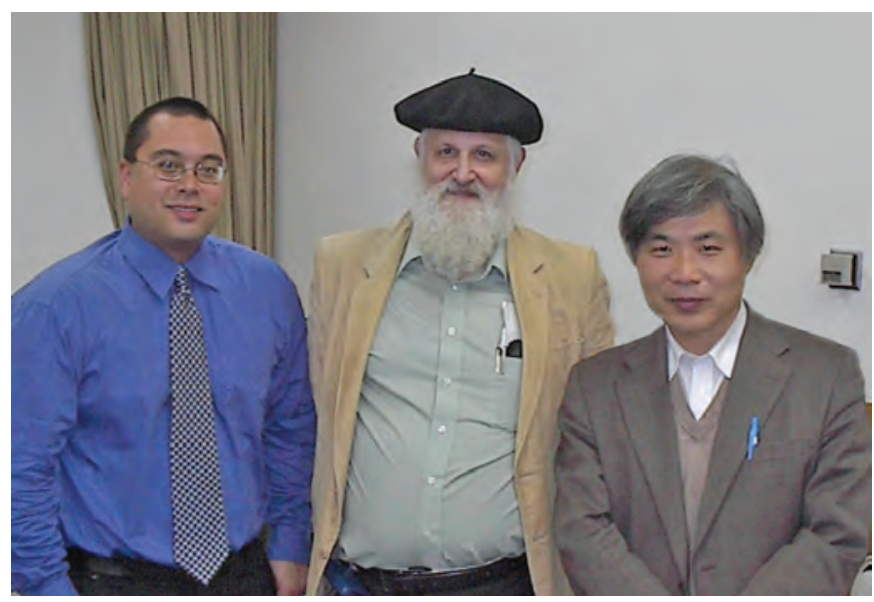

David Damanik, Barry Simon, and Shinichi Kotani, Kyoto, Japan, 2006.

well, that is, whether they are absolutely continuous on $[0, \infty)$ and have only some additional point masses. Since the isolated points below zero will always correspond to point masses, the interesting question is about the nature of the spectral measures on $[0, \infty)$. It was already well known at the time that power decay with $\gamma>1$ implies pure absolute continuity on $(0, \infty)$, that power decay with $\gamma=1$ allows for eigenvalues inside $(0, \infty)$, and that power decay with $\gamma \leq \frac{1}{2}$ allows for disappearance of the absolutely continuous components of the spectral measures. Thus the central questions concerned the $\gamma$ interval $\left(\frac{1}{2}, 1\right)$ and specifically whether the absolutely continuous part survives in all of $(0, \infty)$ and what type of singular components can occur in this energy region.

When I arrived at Caltech, these questions were the most pressing ones in Barry's group. One of his many superb students, Alexander Kiselev, had written his 1997 $\mathrm{PhD}$ thesis on this problem and was successively able to weaken the assumption on $\gamma$ that ensured the survival of the absolutely continuous spectrum on $(0, \infty)$. At the time, the best result used the assumption $\gamma>\frac{2}{3}$, but everyone was betting on $\gamma>\frac{1}{2}$ being sufficient, so that on a power scale there is indeed a sharp transition from the presence of an absolutely continuous spectrum to the possibility of its disappearance at $\gamma=\frac{1}{2}$. This was the so-called “ $\frac{1}{2}$-conjecture." For a class of random decaying potentials, this spectral transition phenomenon was elucidated from a new angle, via modified Prüfer and EFGP transforms, in a 1998 paper Barry wrote together with Alexander Kiselev and Yoram Last.

In one of the major events in spectral theory in the 1990s, the $\frac{1}{2}$-conjecture was proved in 1997 simultaneously, using different methods, by Alexander Kiselev together with Michael Christ, and by Christian Remling, who was another German in Barry's group in 1996-97. In fact, a stronger result was shown that is interesting in its own right: for Lebesgue almost all $E \in(0, \infty)$, all solutions $u$ of $-u^{\prime \prime}(x)+V(x) u(x)=E u(x)$ are bounded.

Recall that the smallness of $V$ near infinity can be expressed through power decay bounds, as well as $L^{p}$ integrability statements. Given the results from the powerdecaying case, one could reasonably conjecture that a 
similar transition in spectral behavior takes place on the $L^{p}$-scale at $p=2$. So Barry, with his never-ending supply of hypertalented students, suggested to Rowan Killip (who had started his graduate studies at Caltech in 1996) that he look at this question. It took only a very short visit of Percy Deift to Caltech for this problem to fall. In the 1999 paper by Deift and Killip, a very slick proof of $V \in L^{2}$ implying absolutely continuous spectrum on $(0, \infty)$ was published. Killip would then go on to extend this result to periodic background and produce another outstanding thesis coming out of Barry's group on the case of decaying potentials, closing out the previous century in style.

With this spectral transition clarified, the other interesting question concerned the nature of the singular spectrum that may be embedded in $[0, \infty)$. For example, can there ever be an embedded singular continuous spectrum, and, if so, under which assumptions on $V$ can it occur? It turned out that the answer to these questions was already implicitly contained in the approach to the first question used by Deift and Killip in terms of a more sophisticated use of sum rules. In two major events at the start of this century this was uncovered. In the process of uncovering what was really going on, Barry was naturally led to learning the history of OPUC and examining in detail the very close connections between OPUC and the theory of Schrödinger operators, which would then keep him busy for a number of years.

First was a 2001 preprint of Serguei Denissov, who used Krein systems to construct embedded singular continuous spectra for some $L^{2}$ potentials. Krein systems are continuum analogs of OPUC, and, in understanding Denissov's preprint, Killip and Simon were prompted to learn new material to understand his proof, which in turn exposed them to the world of OPUC and the realization that the heart of the matter lay in an OPUC result from the early part of the previous century that puts $L^{2}$ decay in 1-1 correspondence with a class of spectral measures.

Second, in the seminal 2003 Killip-Simon paper, quite possibly one of Barry's most influential papers ever, the

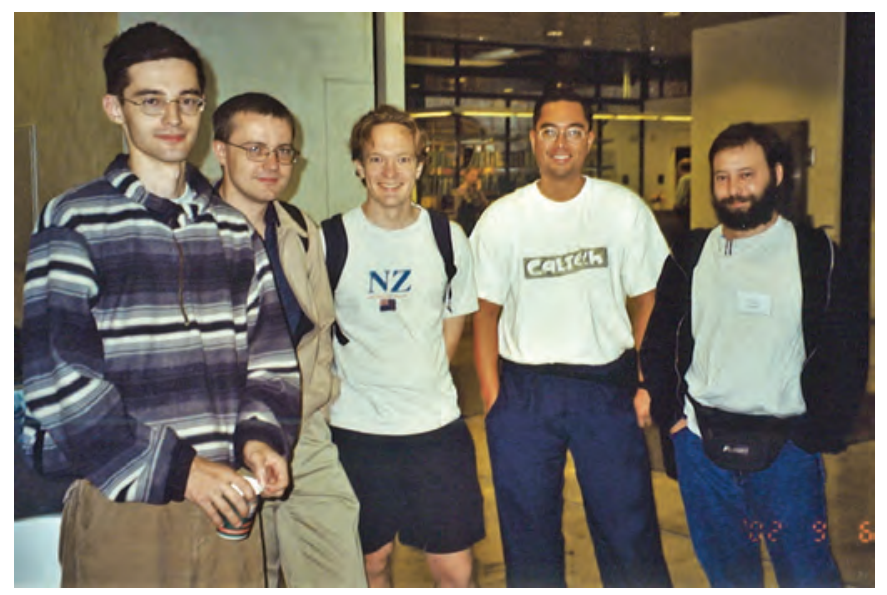

Serguei Denisov, Alexander Kiselev, Rowan Killip, David Damanik, Yoram Last, 2002.

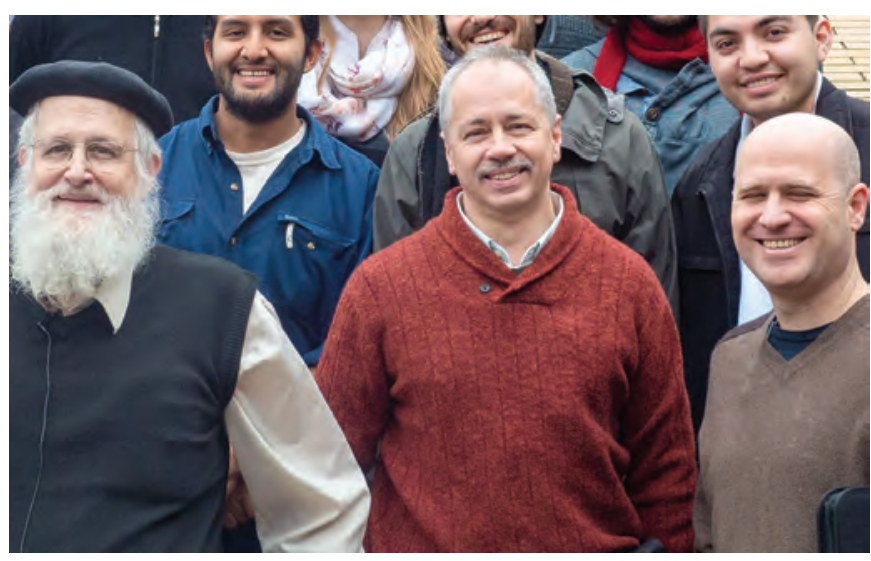

Barry Simon, Andrei Martínez-Finkelshtein, and Jonathan Breuer at Aarhus University, Denmark, 2014.

Jacobi matrix analog of this result was worked out. Semiinfinite Jacobi matrices are in many ways the discrete analog of Schrödinger operators on the half-line, and as a consequence of this result, one could clearly see that $L^{2}$ decay not only allows the occurrence of embedded singular continuous spectra but also puts hardly any restrictions on the kind of embedded singular continuous spectra that can occur. The Schrödinger operator analog appeared later in a 2009 Killip-Simon paper, but the main thrust of the activity in Barry's group following the 2003 Killip-Simon paper was focused on digging into the existing OPUC literature, clarifying what else it may teach us about Schrödinger operators and Jacobi matrices, and, more importantly, revolutionizing the OPUC theory by introducing tools and ideas from the spectral analysis of the latter two classes of operators - and in essence paying back the favor.

In retrospect, the Killip-Simon papers laid bare what the Deift-Killip paper had only hinted at, namely, that the use of sum rules may connect coefficient/potential information to spectral information and that this is in fact a two-way street. This realization is what ushered in the new century in the mathematical physics group at Caltech and prompted Barry to move from exotic spectra to orthogonal polynomials.

\section{Jonathan Breuer and Yoram Last}

\section{Barry between Caltech and Jerusalem}

We both consider ourselves (with pride) to be students of Barry Simon, although formally this is true of neither of us. Aside from his books and papers, which were the basic texts in our graduate education, he mentored us both as postdocs, and we have collaborated, both jointly and separately, with Barry. Each paper we have written with him has been a significant learning experience, and

Jonathan Breuer is associate professor of mathematics at Hebrew University. His email address is jbreuer@math. huj i . ac. 11.

Yoram Last is professor of mathematics at Hebrew University. His email address is y1ast@math. huji.ac. $i 1$. 


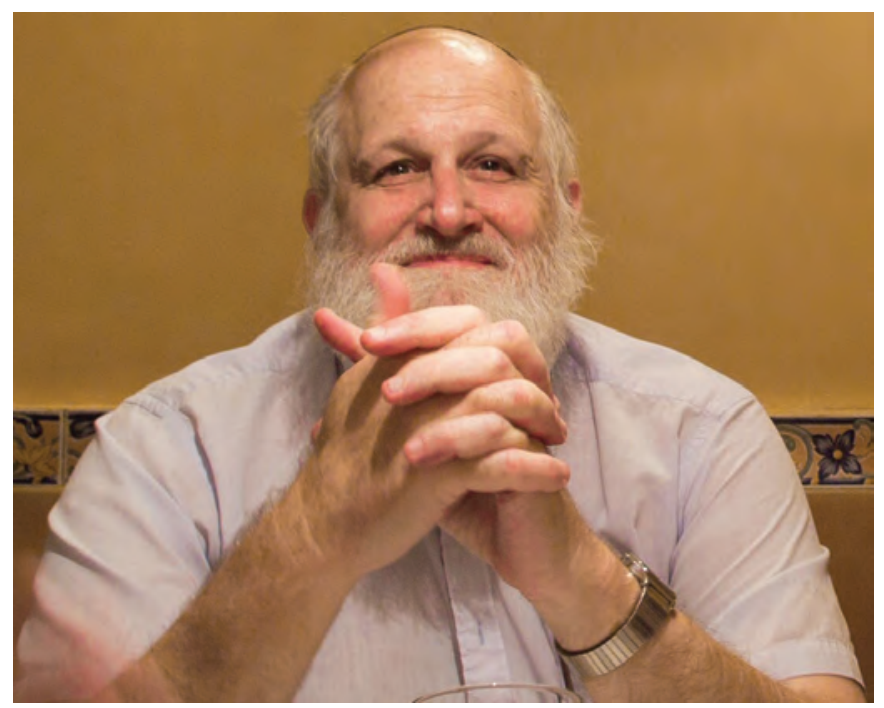

Madrid, 2008.

our two joint collaborations with Barry, both dealing with asymptotics of Christoffel-Darboux (CD) kernels, are no exception.

The first project was started as one of us (JB) was just starting out as a postdoctoral scholar at Caltech with Barry as host. Barry was then writing his book on Szegő's theorem [3] and during a visit to Israel told us about Nevai's delta convergence theorem and its connection to subexponential growth of generalized eigenfunctions of Jacobi matrices. A discussion with one of us (YL) over lunch made it clear that examples could be constructed of regular measures (i.e., models with subexponential growth) for which the delta convergence fails. The job of filling in the details for the construction naturally fell to the most junior member (JB).

JB: “As I arrived at Caltech I was concentrating on filling in the details for this example. However, my wife and I had made a promise to our son that when we got to California we'd go visit Mickey Mouse in Disneyland as soon as we could. Cherie Galvez, Barry's late (and great!) secretary, suggested that since it was September and the academic year had not yet started, we go there on a weekday and not over the weekend when it's crowded, a suggestion we gladly followed. As I walked into Barry's office the following week, however, Barry looked sternly at me and said he was given to understand I had skipped a work day to go to Disneyland. As I was stuttering my response, his stern look became a devilish

\section{This was my first, but not last, encounter with Barry's mischievous side...}

smile and he told me not to worry. Whatever Cherie approves is fine with him. This was my first, but not last, encounter with Barry's mischievous side and my introduction to the positive work atmosphere he creates."
Eventually the paper [1] grew to be much more than a counterexample. We realized that the eigenfunction growth condition was in fact equivalent to the deltaconvergence, extended Nevai's theorem, and made several conjectures. Throughout this project Barry was the clear leader. He formulated the problems, had the best understanding of the context, realized the possible extension of the theorem, and eventually wrote up the results. This is not uncommon with projects where he is involved. However, there are exceptions, one of which is our second joint paper with him.

This paper [2] deals with stability of the convergence of the CD kernel to the sine kernel, and in this case the motivation came more from our side. The general motivating problem behind this paper, which we consider important and largely unsolved, is that of stability of asymptotic level spacing for Schrödinger operators under decaying perturbations. There is an extensive body of literature on the stability of spectral properties. However, almost none of it deals with this type of "fine" spectral property.

One of the theorems in this paper is an illustration of why it is beneficial to get Barry interested in a problem. This theorem says that universality at a point implies that this point is not an eigenvalue of the Jacobi matrix. After thinking about this problem for a little while, we presented it to Barry at lunch on the first day of one of his visits to Jerusalem. By the end of lunch he had a clear and elegant proof and even thanked us for asking the question.

Barry's mathematical prowess, his speed, his depth of insight, his unique ability to see directly to the heart of a problem or a proof are well known to his collaborators and have become legendary through their stories. Slightly less discussed, perhaps, is Barry's leadership and, in particular, his dedication to the advancement of the mathematical fields with which he is associated. The following story is an example.

The ninth OPSFA (orthogonal polynomials, special functions, and applications) international conference took place in Marseille in July 2007. Two remarkable results that were obtained just prior to the start of the conference were Lubinsky's theorem on universal limits of Christoffel-Darboux kernels and Remling's theorem on right limits of Jacobi matrices with absolutely continuous spectrum. Neither Lubinsky nor Remling was speaking at this conference. Nevertheless, Barry, who was a plenary speaker there, felt these two works had to be made known to the community. He thus asked the organizers for two extra slots to discuss these results. Even though the allotted slots were after the end of the daily schedule, both talks were very well attended and were clear and fascinating. There aren't many mathematicians who will volunteer to give two extra talks in a conference on results that aren't even theirs. Barry is not only a scholar but a true leader in his field.

Congratulations Barry on this well-deserved honor. We wish you (and ourselves) many more years of fruitful collaboration. 


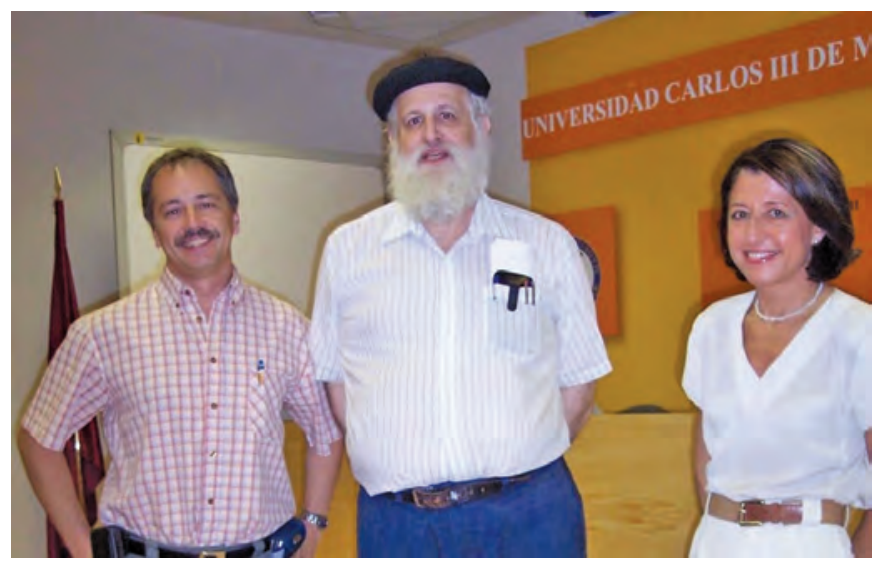

Andrei Martínez-Finkelshtein, Barry Simon, and Maria Jose Cantero, Madrid, 2005.

\section{References}

[1] J. BReuER, Y. LAST, and B. Simon, The Nevai condition, Constr. Approx. 32 (2010), 221-254. MR2677881

[2] _ Stability of asymptotics of Christoffel-Darboux kernels, Commun. Math. Phys. 330 (2014), 1155-1178. MR3227509

[3] B. Simon, Szegó's Theorem and Its Descendants: Spectral Theory for $L^{2}$ Perturbations of Orthogonal Polynomials, M. B. Porter Lectures, Princeton University Press, Princeton, NJ, 2011. MR2743058

\section{Andrei Martínez-Finkelshtein}

\section{Orthogonal Polynomials and Spectral Theory: Barry's Revolution}

Qualifying exams were tough at Moscow State University, at least at the end of the 1980s. Those in analysis consisted of real and complex analysis, harmonic analysis, and operator and spectral theory. In other words, basically the content of [2]. Looking for good textbooks, I was advised to read the first two volumes of the Russian translation of [1]. Books, especially scientific books, were cheap in the Soviet Union, affordable even by a graduate student, so I went to a bookstore to get my own copy. All volumes were out of print. Fortunately, there was a welldeveloped network of "Bukinists," used bookstores where I found all volumes except the most important one for me, Volume I, Functional Analysis. I checked unsuccessfully in several places, leaving the $n$-th Bukinist disappointed, when I was called by a mysterious guy who in a low voice offered me the desired Volume I for several times its official price! Indeed, Moscow at that time was a curious place, where smugglers made profit from Reed \& Simon. Thus, my first and indirect encounter with Barry was not deprived of a certain excitement. When much later I heard that Barry had written a paper on orthogonal polynomials, I could not believe that it was the very same Barry Simon! I learned later how young Barry was when he wrote [1].

The role of Barry in the rapid development of the theory of orthogonal polynomials in the last twenty years,

Andrei Martínez-Finkelshtein is professor of applied mathematics at the University of Almería. His email address is andrei@ua1 . es . especially in the use of spectral theory techniques, is well known and documented. This exemplifies the often described and admired feature of Barry: how fast he can work. I think it was very early in 2004 when I received a message from Barry asking me to take a look at a paper. Before I had time to read it carefully, the small paper grew into a much bigger one, and I got an updated version, which had the same fate. Days (I mean DAYS) later the paper became a short book, then a longer book, then in April 2004 he sent the message:

It's done!! It's done!! Well sort of. I have "essentially" completed my book on Orthogonal Polynomials on the Unit Circle.

Obviously, the book didn't stop growing, with about biweekly updates, until it was published in two volumes and more than one thousand pages!

It contained both classical and new results in orthogonal polynomials, spectral theory, and complex analysis. For instance, it showed the central role played by the matrices, related to OPUC in the same way as Jacobi matrices are related to orthogonal polynomials on the real line.

There were also higher-order analogues of Szegő's theorem, that is, conditions on integrability of expressions containing the logarithm of the orthogonality weight in terms of the recurrence (or Verblunsky) coefficients of the corresponding orthogonal polynomials.

About ten years after its publication, Orthogonal Polynomials on the Unit Circle is one of the most outstanding contributions to the field, both in terms of scientific impact and popularity, an indispensable reference for researchers, comparable to the influence that the classical monograph of Szegó [3] had in its time. It also stimulated a burst of activity in the area: "If Barry Simon is interested in orthogonal polynomials, there should be something in it!"

Barry and his collaborators also made numerous contributions to the theory of orthogonal polynomials on the real line, especially at the boundary with spectral theory.

Orthogonal polynomials on the real line are characterized by their three-term recurrence relations, whose coefficients can be assembled into a three-diagonal (Jacobi) matrix $J$. The spectral measure of this semi-infinite matrix is precisely the orthogonality measure for the polynomials. Simon and his collaborators made very significant contributions to both direct

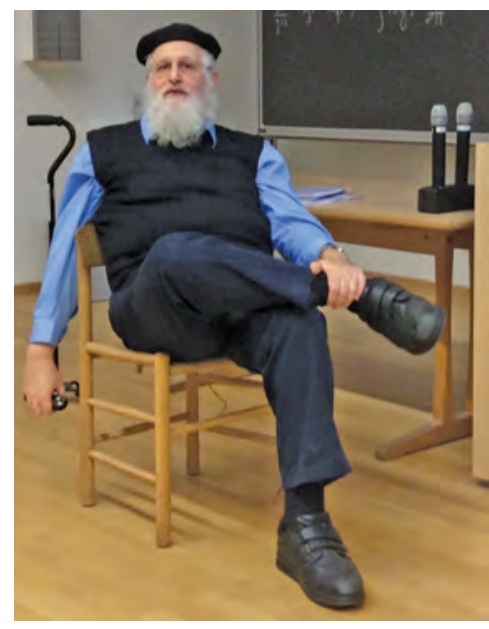

Aarhus University, Denmark, 2014. (which try to read the properties of this measure from the behavior of the entries of the Jacobi matrix) and inverse spectral problems. For instance, they characterized when 
$J$ is an $\ell^{2}$ perturbation of either a "free" or a periodic Jacobi matrix, or they found higher-order analogues of the Szegó condition, developing for that purpose several useful technical tools.

Zeros of orthogonal polynomials have independent interest and applications, and their behavior is being actively studied. Their fine structure is strongly connected to the so-called universality behavior of the ChristoffelDarboux kernels of the associated polynomials, relevant to statistics of eigenvalues of random matrices, a subject on which there is an enormous amount of discussion in both the mathematics and the physics literature. Avila, Last, and Simon showed in 2010 that universality and the so-called "clock behavior" of zeros on the real line in the absolutely continuous spectral region is implied by convergence for the diagonal Christoffel-Darboux kernel and by boundedness of its analogue associated with second kind polynomials. They also showed that these hypotheses are always valid for ergodic Jacobi matrices with absolutely continuous spectra.

I am also interested in asymptotic problems for orthogonal polynomials, and during 2004-05 some of my work overlapped with Barry's research; together with Ken McLaughlin and Ed Saff we were focusing on the asymptotics of orthogonal polynomials on the unit circle with respect to analytic weights. The zeros of such polynomials were also, and almost simultaneously, studied by Simon. But our techniques were very different: While we were using the newly created tool of Riemann-Hilbert analysis, Simon's approach was more classical, obviously borrowing ideas from spectral theory. At a conference in honor of Percy Deift's birthday, Barry referred to the Riemann-Hilbert method (which yields impressive results but invariably requires lengthy calculations) as "driving a Caterpillar truck," as opposed to his "using an ax" in order to open a path through the jungle of the unknown towards the desired goal. Later, looking at the exhaustive

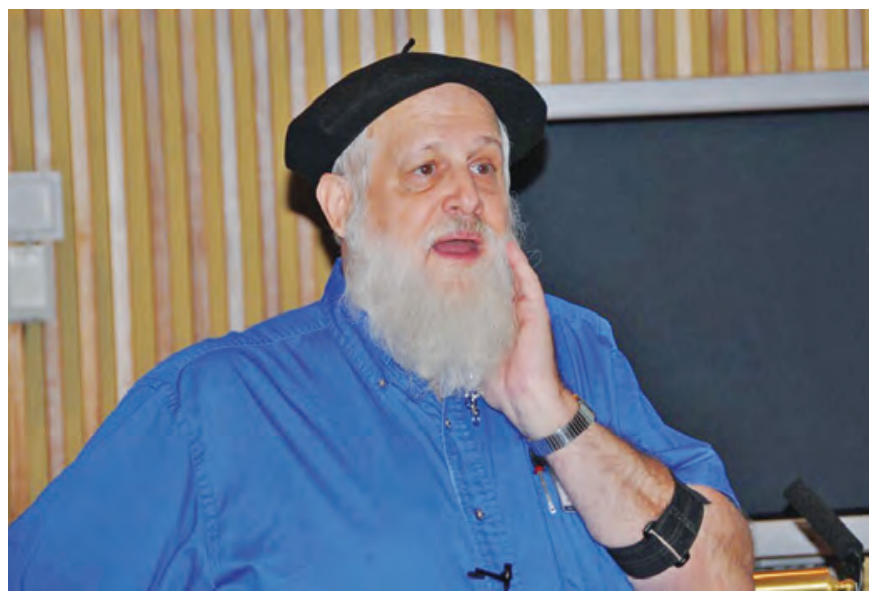

Madrid, 2008.

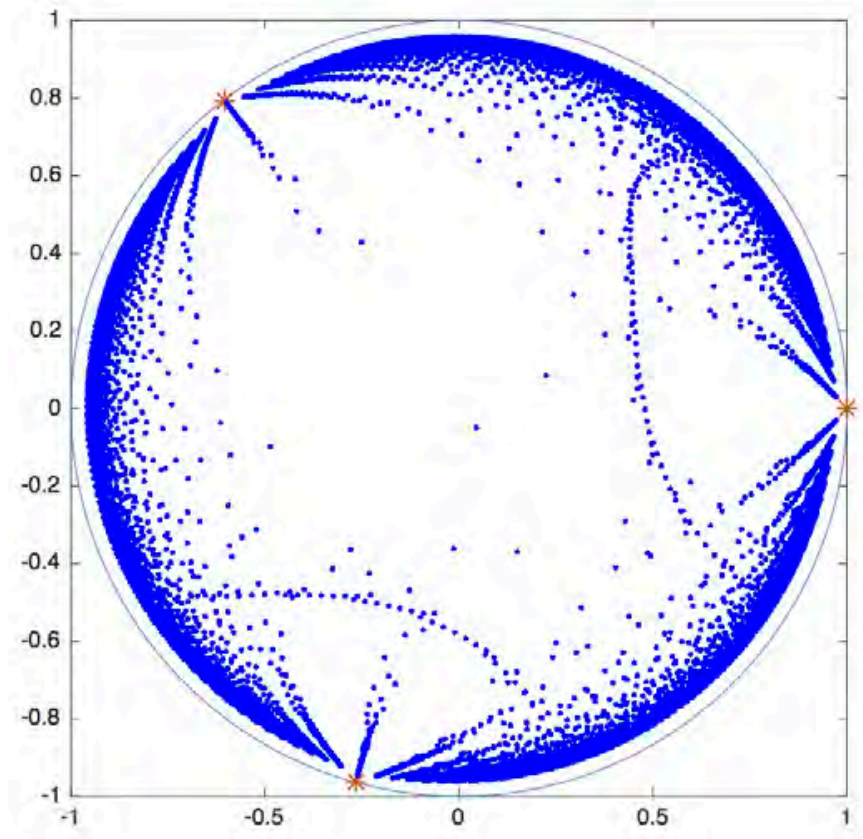

Zeros of orthogonal polynomials on the unit circle of degrees $n=1,2, \ldots, 150$, with respect to the weight $W(z)=|z-1|^{1 / 5}|z-a|^{-2 / 5}\left|z-a^{2}\right|^{-1 / 5}$, for $|z|=1$, with $a=\exp (\pi i \sqrt{2} / 2)$.

results that Barry was able to obtain, I compared his method to using not an ax, but napalm. ${ }^{2}$

Due to our mutual interest in these topics, I got an invitation to visit Barry at Caltech in 2008 and was able to see him in action. I actually visited Caltech several times, with almost a year's spacing. These visits were highly enjoyable and stressful at the same time. I felt like a graduate student, and although it was a test for my selfesteem, it was a fantastic experience. We started to work, but it progressed slowly, partially due to my distraction with so many other things I had to learn from Barry. Our typical interaction, on the rare occasions when it was I who came up with a new idea, was like this: After spending the whole weekend immersed in lengthy computations, I would ask Cherie, ${ }^{3}$ Barry's long-time secretary, for an appointment to see him, and I would proudly scribble my formulas on the blackboard. In the event
"Barry writes books in the time others write papers" they were right, Barry would look at them for a while in silence, slightly squinting and playing with his beard, then murmur that it was a bit late, that he needed to drive

${ }^{2}$ This controversy between Caterpillar truck and ax went on for a while. Barry has a notorious sense of humor, often reflected in his writing.

${ }^{3}$ Sadly, Cherie passed away in July of 2013. 


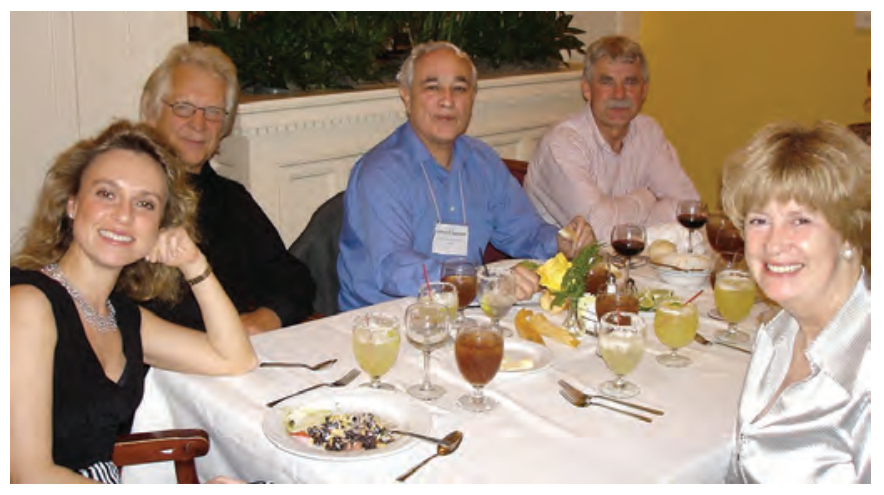

Olga Holtz, Herbert Stahl, Guillermo LópezLagomasino, Vilmos Totik, and Kathy Driver, San Antonio, 2010.

home, but that he thought he could prove it in a few lines. An hour later (about the time it would take him to drive home from Caltech!) I would receive a scan of Barry's "doctor handwriting" containing a proof...in a few lines!

Here are a few more observations about Barry from that time:

- Barry has a vast culture. Not only does his personal toolbox contain so many mathematical results, theories, formulas, and ideas, but he masterfully applies them elsewhere. He has quite wide interests: computers and politics, just to mention two of them. He knows a lot about these topics and discusses them with passion. A preferred place for such discussions was the so-called "brown bag meetings" at Caltech, right after his seminars. One day Barry was regretting that he was spending too much time following political news, and I wondered what more he could have done without "wasting" this time.

- Barry is so fast it sometimes looks unreal. I already told how he would re-prove my laboriously obtained results when driving home. But I witnessed how he would "spoil" somebody's punchline at a seminar talk, exclaiming a few minutes into the talk, "Ah, you are going to do this and this, claiming that...!"

- On top of this, Barry is extraordinarily well organized. I mentioned that everybody visiting Barry needed an appointment to meet him, and his schedule was strictly respected.

All these factors sum up to Barry's legendary productivity: his five-volume Comprehensive Course in Analysis has 3,259 pages! Quoting Vilmos Totik, "Barry writes books in the time others write papers."

I will finish by mentioning Barry Simon's teaching, which has had a tremendous impact on the community. His lectures and review papers have had a great influence on numerous people in a wide range of fields in physics and mathematics and have served as an enormous source of inspiration. Barry is a passionate lecturer who masters the blackboard, something not so common in these days of multimedia presentations. I remember that in June of 2005 Barry gave a two-day seminar at the University Carlos III de Madrid. It was bad timing: the main lecture halls were closed for some reason, and we had to squeeze into a small room with a tiny board, about $5 \times 7$ feet! We were all rather concerned about Barry's reaction, but he masterfully gave the whole course, using every single one of those 35 square feet.

In contrast with that, the 9th International Symposium on Orthogonal Polynomials, Special Functions, and Applications took place in Marseille two years later, and Barry volunteered to give an extra late-evening session on some hot topics on orthogonal polynomials. The main lecture room in the International Center for Mathematical Meetings of the French Mathematical Society in Luminy was spectacular, the blackboard made of nine large moving panels. The use of this surface by Barry was masterful again; all blackboards were filled with formulas and theorems, going up and down in front of the audience in an impressive exhibition of his communication skills.

Throughout his scientific career Barry Simon has had a special concern for young (and not so young) scientists. The long list of people who have worked with Barry Simon is remarkable and includes many $\mathrm{PhD}$ students, postdoctoral fellows, and collaborators from many fields. It is a privilege and an honor for me to be part of this list. There are still several open questions and unfinished projects with Barry, and I hope to be able to ask him for an appointment again in the near future and to scribble my formulas on a blackboard, even risking to hear from him, "I think I can prove it in a few lines!"

\section{References}

[1] M. REED and B. SIMON, Methods of Modern Mathematical Physics, Vols. I-IV, Academic Press, New York, 1st ed., 1972-1979. MR0493419 MR0493420, MR0493421, MR0529429

[2] B. Simon, A Comprehensive Course in Analysis, Vols. 15, Amer. Math. Soc., Providence. RI, 2015. MR3408971, MR3443339, MR3364090, MR3410783

[3] G. SzEGó, Orthogonal Polynomials, 4th ed., AMS Colloq. Publ., Vol. 23, Amer. Math. Soc., Providence, RI, 1975. MR0372517

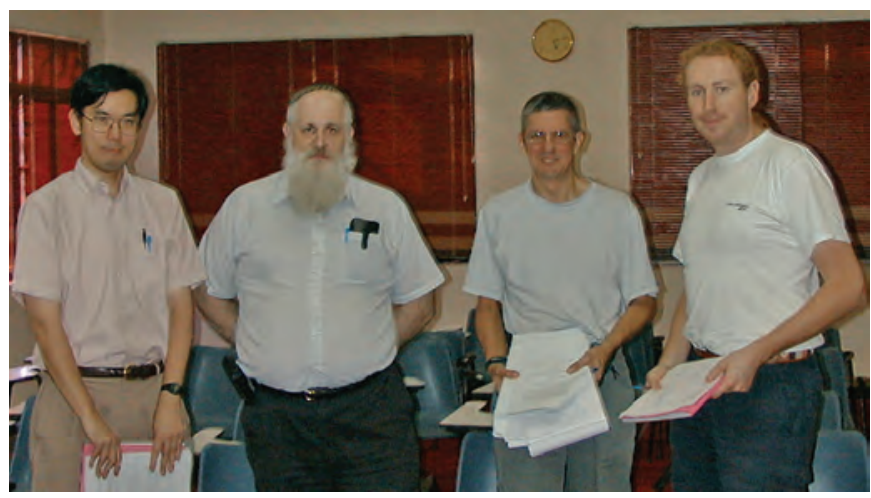

Shu Nakamura, Barry Simon, Peter Hislop, and Frédéric Klopp, Goa, India, 2000. 


\section{Credits}

Page 2 photo of Barry and Yosi, courtesy of Barry Simon. Page 3 photo of Martha and Barry, courtesy of Heinrich Steinlein.

Figure 2, courtesy of Yosi Avron and Daniel Osadchy.

Page 5 head shot, courtesy of Svetlana Jitomirskaya.

Page 5 photo of Simon in Marseille, courtesy of Andrei Martínez-Finkelshtein.

Page 6 illustration, courtesy of Barry Simon.

Photos of Barry in Japan, and 2002 students and postdocs, courtesy of David Damanik.

Page 8 photo of Barry in Denmark, 2014 and pages 9-11 photos and artwork, courtesy of Andrei MartínezFinkelshtein.

Page 12 photo taken in San Antonio, 2010, courtesy of Vilmos Totik.

Page 12 photo taken in Goa, India, 2000, courtesy of Barry Simon.

\section{Twenty Years Ago in the Notices}

September 1996

Finsler Geometry Is Just Riemannian Geometry without the Quadratic Restriction, by Shiing-Shen Chern.

The outstanding mathematician explains why Finsler geometry is a natural setting for Riemannian geometry in many and diverse situations.

www.ams.org/notices/199609/ chern.pdf 